\title{
Optimal weighting models based on linear uncertain constraints in intuitionistic fuzzy preference relations
}

\author{
Zaiwu Gong ${ }^{1 *}$, Xiao Tan ${ }^{1}$ and Yingjie Yang $^{2}$ \\ ${ }^{1}$ Collaborative Innovation Center on Forecast and Evaluation of Meteorological Disasters, \\ School of Management Science and Engineering, \\ Nanjing University of Information Science and Technology, Nanjing, China \\ ${ }^{2}$ Centre for Computational Intelligence, De Montfort University, Leicester, UK \\ *Corresponding Author Email: zwgong26@163.com
}

\begin{abstract}
The priority weight vectors of an intuitionistic fuzzy preference relation (IFPR) with linear uncertainty distribution characteristics in group decision making (GDM) are determined in this study. On the basis of an IFPR, the assumptions of additive consistency and decision-making preference variables obeying the uncertainty distribution are defined. Afterwards, a priority model is constructed with a chance constraint, and the ranking relations of the membership and non-membership matrices are analysed. The change in the confidence level of the chance constraint controls the flexibility of realizing additive consistency. Moreover, it is proven that if the individual decision makers' IFPR has a linear distribution, the group IFPR aggregated by the weighted methodology still obeys this distribution. Finally, an uncertain linear ranking consensus model of the IFPR is developed, and a numerical example is used to verify its feasibility.
\end{abstract}

Keywords: group decision making; intuitionistic fuzzy preference relation; chance constraint; uncertainty distribution; additive consistency; uncertain programming 


\section{Introduction}

Group decision making (GDM) refers to the alternatives (criteria, properties, and schemes) judgment and GDM behaviour. Generally, the common solution achieved by the consensus process can satisfy most of the expectations and preferences of the decision makers (DMs), and concrete priority ranking makes the consensus more complete. Owing to the complexity of and uncertainty in decision environments and the DMs' behavioural choices, there is a great possibility that DMs cannot provide a precise and exact judgment scheme. The fuzzy theory proposed by Zadeh (1965) overcomes the limitation of accurate judgment well. DMs prefer using pairwise comparisons (PCs) in fuzzy situations, which are expressed in the form of a preference relation matrix. This relation defines the fuzziness and uncertainty in different formats such as the interval fuzzy preference relation (Meng, An, \& Chen, 2016; Gong et al., 2015), linguistic fuzzy preference relation (Jin, Ni, Chen, \& Li, 2016b; Zhao, Ma, \& Wei, 2017), IFPR (Zhang \& Guo, 2017; Zhu \& Xu, 2013), triangular fuzzy preference relation (Liu, Zhang, \& Zhang, 2014; Wang \& Tong, 2016), and hesitant fuzzy preference relation (Zhang, Z., 2016; Zhu \& Xu, 2013). Unreasonable PCs will contribute to unacceptable consensus results, which means that it is necessary to guarantee that PCs meet the consistency level. Therefore, measurement of the decision consistency is a critical issue in GDM; it can check the logic of DMs and then estimate whether or not the (group) conclusion is rational. To recapitulate, dealing with preference relations, obtaining the priority weights, enhancing consistency, reaching a consensus, and selecting alternatives are key points in GDM (Chu, Liu, Wang, \& Qin, 2016; Dong, Li, Chiclana, \& Herrera-Viedma, 2016; Gong, Zhang, Forrest, Li, \& Xu, 2015; Jin, Ni, Chen, \& Li, 2016a; Meng et al., 2016).

Atanassov (1986) extended fuzzy theory and proposed an IFPR to describe the vagueness in real life more effectively and accurately, and the advantage of an IFPR lies in representing inevitably imprecise or not totally reliable judgments (Behret, 2014). Comprehensively dealing with membership, non-membership and hesitancy, IFPRs simultaneously can translate an intuitionistic fuzzy matrix into two equivalent interval matrices: membership and non-membership matrices (Gong, 2011). In the process of handling incomplete fuzzy preference relations, existing models (Meng et al., 2016; Wang \& Li, 2015; Wu \& Chiclana, 2014; Zhang, Wang, \& Tian, 2015) were used to study the inherent properties of IFPRs, interval fuzzy preference relations, and hesitant fuzzy preference relations to estimate missing values. In order to obtain a priority ranking and consensus results, Wu and Chiclana (2014), and Zhang and Guo (2017) measured the consensus and defined operators to grasp the characteristic information of an individual IFPR and the collective IFPR, whereas Jin et al. (2016a), Wang (2013), Wang, Lan, Ren, \& Luo (2012), and Zhang, Z. (2016) constructed goal programming models to derive weight vectors. Liao, Xu, Zeng, \& Xu (2016) improved a model that removes the expert from the decision 
group to avoid the loss of valuable information. Many studies have designed weighted operators (Ouyang \& Pedrycz, 2016; Tan, Yi, \& Chen, 2015; Wang \& Li, 2015) and different algorithms (Chu, 2016; Jin et al., 2016b; Wang, 2015; Xu \& Xia, 2014) on the basis of various preference relations to reach a consensus.

Consistency is an effective instrument for judging whether or not the decision information is reasonable. An analysis of the consistency is necessary in the process of decision checking; it can test if the judgments aimed at multiple alternatives are in accordance with mathematical logic principles. The purpose of studying an individual's consistency is to evaluate an individual DMs' judgment ability and to obtain priority weights. The purpose of studying the collective consistency is to estimate the consensus. Studies of consistency are mostly based on the additive-transitivity and multiplicative-transitivity properties Tanino (1984) defined and developed respective applicable consistency definitions aiming at different preference relations (Chen, Cheng, \& Lin, 2015; Jin et al., 2016a; Jin et al., 2016b; Krejčí, 2017; Liu, 2014; Meng et al., 2016; Wang \& Tong, 2016; Wu \& Chiclana, 2014; Wu \& Xu, 2016; Xu \& Xia, 2014; Zhang, HM., 2016; Zhang, Z., 2016; Zhang \& Guo, 2017; Zhao et al., 2017). Chen, Lin, \& Lee (2014) applied additive consistency to estimate missing fuzzy numbers and hesitant numbers. Dong et al. (2016) measured the average-case consistency and modified interval-valued reciprocal preference relations based on the additive consistency. Krejčí (2017) defined two types of additive consistency to avoid violating the reciprocity of PCs, and Jin et al. (2016b) probed the linguistic expression, introduced order consistency and additive consistency, and then measured whether the preference relation has an acceptable additive consistency by a consistency index. Moreover, the multiplicative consistency has been investigated (Jin et al., 2016a; Liu et al, 2016; Wu \& Chiclana, 2014; Xu \& Xia, 2014; Zhang, 2015; Zhang, HM., 2016; Zhang, Z., 2016). Because the IFPR can be transformed into interval fuzzy preference relations after the consistent IFPR proposed by Xu (2007), consistency is increasingly applied to IFPRs. Recent studies include those by Chu et al. (2016), Jin et al. (2016a) and Zhang \& Guo (2017), which constructed consistency definitions to modify the rationality of the PCs of objects. Wu and Chiclana (2014) also dealt with the missing information in an IFPR, quantitatively proposed an acceptable consistency index, and provided new definitions. Behret (2014) improved the consistency level by minimizing the deviations from the additive and multiplicative consistency perspective respectively.

Mathematical programming models have been widely applied to priority ranking based on the consensus in GDM. For example, Zhang, Z. (2016) derived the priority weights by combining incomplete preference relations and the multiplicative consistency linearly; Liao and Xu (2014), Wang et al. (2012), Xu et al. (2014) and Zhang, HM. (2016) noted multiplicative and additive characteristics; 
and Behret (2014) constructed linear and nonlinear programming models considering the additive and multiplicative consistency, respectively. In addition, the optimal deviation values between any provided IFPR and the converted multiplicative consistent IFPR obtained from a programming model can improve the consistency (Jin et al., 2016a).

In the situation considered in this paper, the constraints in the programming model contain random variables, and DMs should make a decision before recognizing the realization of these variables. Considering that the constraints may not be satisfied under adverse situations, the following principle adopted: allow the decision making not to meet the constraint conditions to some extent, but the probability of a tenable constraint condition is no less than a certain confidence level (Liu \& Zhao, 1998; Tan, Gong, Chiclana, \& Zhang, 2017; Zhang, Gong, \& Chiclana, 2017). This goal programming is called chance-constrained programming.

There are two methods for solving chance-constrained programming: one is approaching the effective solution to decision problems by a stochastic simulation or an intelligent algorithm (Ke \& Liu, 2007; Suo, Li, Wang, \& Yu, 2017), and the other is transforming it into an equivalent programming problem, such as introducing a goal programming model (Omidi, Abbasi, \& Nazemi, 2017). In this paper, we assume that the IFPR is equivalent to interval fuzzy preference relations, and the interval is presented in the form of a range with lower and upper limits. Therefore, we may be able to view the judgments as uncertain random variables obeying a uniform uncertainty distribution (the normal distribution and other distributions are also feasible (Liu, 2015)) in the interval. Then, for a certain confidence level, the deviation between the judgment of each DM and the ideal judgment (satisfying consistency) should be minimized. Therefore, optimal weighting models based on the uncertain constraints in the IFPR are constructed, and the optimal solution for decision making is achieved by an equivalent goal programming model.

The paper is organized as follows. The next section reviews several related basic concepts of the fuzzy preference relation, interval fuzzy preference relation, and IFPR and investigates the relation between the IFPR and the interval fuzzy preference relation. In the following section, the definition of an IFPR with a linear uncertainty distribution and its consistency concept are introduced. Then, the next section presents the individual IFPR and its collective optimal priority weight vector, and the relation between membership and non-membership is investigated. Next, a numerical example of this new model is presented, and the conclusions and future plans are discussed in the last section. 


\section{Preliminaries}

The following sets are defined: $N=\{1,2, \cdots, n\}, M=\{1,2, \cdots, m\}$. Let $X=\left\{x_{1}, x_{2}, \cdots, x_{n}\right\}$ be a limited set of alternative judgments, where $x_{i}(i \in N)$ represents the decision-making judgment of alternative $i$. DMs usually adopt PCs to obtain the priority weight vector by constructing different types of fuzzy preference relations such as interval fuzzy preference relations and IFPRs.

\section{Fuzzy preference relations and the corresponding weights}

Definition 1: The matrix $A^{\prime}=\left(a_{i j}^{\prime}\right)_{n \times n}, i, j \in N$ is called a fuzzy preference relation, if

$$
\begin{aligned}
a_{i i}^{\prime} & =0.5, i \in N \\
a_{i j}^{\prime}+a_{j i}^{\prime} & =1, i, j \in N
\end{aligned}
$$

The element $a_{i j}^{\prime}$ in matrix $A^{\prime}$ implies the degree of membership of alternative $x_{i}$ over alternative $x_{j}$. If $a_{i j}^{\prime}=0.5$, there is no difference between $x_{i}$ and $x_{j}$; if $a_{i j}^{\prime}>0.5, x_{i}$ is superior to $x_{j}$; and if $0 \leq a_{i j}^{\prime}<0.5, x_{i}$ is inferior to $x_{j}$.

Definition 2: The fuzzy preference relation $A^{\prime}$ is called an additive consistency fuzzy preference relation, if

$$
a_{i j}^{\prime}+a_{j k}^{\prime}+a_{k i}^{\prime}=1.5, i, j, k \in N
$$

Theorem 1: If the fuzzy preference relation $A^{\prime}=\left(a_{i j}^{\prime}\right)_{n \times n}$ has the weight vector $\omega^{\prime}=\left(\omega_{1}^{\prime}, \omega_{2}^{\prime}, \ldots\right.$, $\left.\omega_{n}^{\prime}\right)^{T}$, that satisfies

$$
a_{i j}^{\prime}=\frac{1}{2}\left(\omega_{i}^{\prime}-\omega_{j}^{\prime}+1\right), i, j \in N
$$

where $\sum_{i=1}^{n} \omega_{i}^{\prime}=1,0 \leq \omega_{i}^{\prime} \leq 1$, then $A^{\prime}$ is an additive consistency fuzzy preference relation.

Proof. Because $a_{i j}^{\prime}+a_{j k}^{\prime}+a_{k i}^{\prime}=\frac{1}{2}\left(\omega_{i}^{\prime}-\omega_{j}^{\prime}+1\right)+\frac{1}{2}\left(\omega_{j}^{\prime}-\omega_{k}^{\prime}+1\right)+\frac{1}{2}\left(\omega_{k}^{\prime}-\omega_{i}^{\prime}+1\right)=1.5$, the matrix $A^{\prime}$ satisfies additive consistency.

This paper adopts the commonly used consistency condition as Theorem 1 proposed by Tanino in 1984. Several scholars discussed this condition by adjusting 0.5 in original mathematical expression to uncertain coefficient (Hu, Ren, Lan, Wang, \& Zheng, 2014; Liu, Pan, Xu, \& Yu, 2012; Wang, 2016; Wang \& Li, 2016). However, constructing priority model with a chance constraint is the emphasis, we still use the original one. The method for determining the priority weights is also applicable to the consistency condition with unknown coefficient.

\section{Interval fuzzy preference relations and the corresponding weights}

A certain judgment $\left(a_{i j}^{\prime}\right.$ is crisp number) is not practical to be given when making PCs in a real decision making situation. In order to achieve a more realistic decision, DMs use a range (namely, an 
interval) instead of a crisp number.

Definition 3: The preference relation $\bar{A}=\left(\bar{a}_{i j}\right)_{n \times n}$ is an interval fuzzy preference relation, where $\bar{a}_{i j}=\left[a_{i j l}, a_{i j u}\right]$, if

$$
\begin{aligned}
\bar{a}_{i i} & =[0.5,0.5], i \in N \\
a_{i j l}+a_{j i u} & =a_{j i l}+a_{i j u}=1, i, j \in N
\end{aligned}
$$

The element $\bar{a}_{i j}=\left[a_{i j l}, a_{i j u}\right]$ in matrix $\bar{A}$ implies the degree of membership of alternative $x_{i}$ over alternative $x_{j}$. If $\bar{a}_{i j}=\left[\bar{a}_{i j l}, \bar{a}_{i j u}\right]=[0.5,0.5]$, there is no difference between $x_{i}$ and $x_{j}$; if $\bar{a}_{i j}>[0.5,0.5]$, $x_{i}$ is superior to $x_{j}$; and if $0 \leq \bar{a}_{i j}<[0.5,0.5], x_{i}$ is inferior to $x_{j}$.

The matrix $\bar{A}=\left(\bar{a}_{i j}\right)_{n \times n}$, where $\bar{a}_{i j}=\left[a_{i j l}, a_{i j u}\right]$, let $\bar{a}_{i j}=\left(1-\theta_{i j}\right) a_{i j l}+\theta_{i j} a_{i j u}$ and $\bar{a}_{j i}=$ $\left(1-\theta_{i j}\right) a_{j i u}+\theta_{i j} a_{j i l}, i<j$ for $0 \leq \theta_{i j} \leq 1, i, j \in N$. Obviously, for any certain coefficient $\theta_{i j}$, there exist $\bar{a}_{i i}=\frac{1}{2}\left(a_{i i l}+a_{i i u}\right)=0.5$ and $\bar{a}_{i j}+\bar{a}_{j i}=\left(1-\theta_{i j}\right) a_{i j l}+\theta_{i j} a_{i j u}+\theta_{i j} a_{j i l}+\left(1-\theta_{i j}\right) a_{j i u}=1, i<j$. Therefore, the interval preference relation $\bar{A}=\left(\bar{a}_{i j}\right)_{n \times n}=\left(\left(1-\theta_{i j}\right) a_{i j l}+\theta_{i j} a_{i j u}\right)_{n \times n}$ can be recognized as a fuzzy preference relation.

Definition 4: $\bar{A}$ is an additive consistency fuzzy preference relation, if there exists $\theta_{i j}, 0 \leq \theta_{i j} \leq 1$, such that

$$
\bar{a}_{i j}+\bar{a}_{j k}+\bar{a}_{k i}=1.5, i, j, k \in N
$$

where $\bar{a}_{i j}=\left(1-\theta_{i j}\right) a_{i j l}+\theta_{i j} a_{i j u}, \bar{a}_{j i}=\theta_{i j} a_{j i l}+\left(1-\theta_{i j}\right) a_{j i u}, i<j$.

Similar to Theorem 1, the weight theorem of an interval fuzzy preference relation is as follows:

Theorem 2: If an interval fuzzy preference relation $\bar{A}=\left(\bar{a}_{i j}\right)_{n \times n}$ has the weight vector $\bar{\omega}=$ $\left(\bar{\omega}_{1}, \bar{\omega}_{2}, \ldots, \bar{\omega}_{n}\right)^{T}$ and coefficient $\theta_{i j}$, and satisfies

$$
\bar{a}_{i j}=\frac{1}{2}\left(\bar{\omega}_{i}-\bar{\omega}_{j}+1\right), i, j \in N
$$

where $\sum_{i=1}^{n} \bar{\omega}_{i}=1,0 \leq \bar{\omega}_{i} \leq 1$, and $\bar{a}_{i j}=\theta_{i j} a_{i j l}+\left(1-\theta_{i j}\right) a_{i j u}, \bar{a}_{j i}=\left(1-\theta_{i j}\right) a_{j i u}+\theta_{i j} a_{j i l}, i<j, 0 \leq$ $\theta_{i j} \leq 1$, then $\bar{A}$ is additive consistent, and $\bar{\omega}$ is called the weight vector of $\bar{A}$.

The proof is omitted.

\section{Intuitionistic fuzzy preference relations}

An IFPR can solve the dilemma that the DMs face when discussing the specific degree to which one alternative is better than others; therefore, the degrees of membership, non-membership, and hesitancy are adopted to express the affirmation, negation, and hesitation shown by DMs (Behret, 2014). Compared with interval judgment, an IFPR is in accordance with the DMs' behavioural characteristics and uncertain decision-making problem. 
Definition 5: $R=\left\{\left\langle\left(x_{i}, x_{j}\right), \mu_{R}\left(x_{i}, x_{j}\right), \nu_{R}\left(x_{i}, x_{j}\right)\right\rangle \mid\left(x_{i}, x_{j}\right) \in X \times X\right\}$ is an IFPR with a universe of discourse $X$, where $\mu_{R}: X \times X \in[0,1], \nu_{R}: X \times X \in[0,1], \mu_{R}: X \times X$ indicates the degree to which $x_{i}$ is superior (inferior) to $x_{j}, \nu_{R}: X \times X$ indicates the degree to which $x_{i}$ is not superior (not inferior) to $x_{j}$, and $0 \leq \mu_{R}\left(x_{i}, x_{j}\right)+\nu_{R}\left(x_{i}, x_{j}\right) \leq 1$ holds.

Definition 6: Let $R$ be an IFPR with a universe of discourse $X$. If for any $i, j \in N, \mu_{i j}=$ $\mu_{R}\left(x_{i}, x_{j}\right), \nu_{i j}=\nu_{R}\left(x_{i}, x_{j}\right)$, such that??

$$
r_{i i}=(0.5,0.5,0) ; \quad \mu_{i j}=\nu_{j i}, \nu_{i j}=\mu_{j i}, \pi_{i j}=\pi_{j i} ; \quad \mu_{i j}+\nu_{i j}+\pi_{i j}=1
$$

then

$$
R=\left(\mu_{i j}, \nu_{i j}, \pi_{i j}\right)_{n \times n}=\left(\begin{array}{cccc}
\left(\mu_{11}, \nu_{11}, \pi_{11}\right) & \left(\mu_{12}, \nu_{12}, \pi_{12}\right) & \cdots & \left(\mu_{1 n}, \nu_{1 n}, \pi_{1 n}\right) \\
\left(\mu_{21}, \nu_{21}, \pi_{21}\right) & \left(\mu_{22}, \nu_{22}, \pi_{22}\right) & \cdots & \left(\mu_{2 n}, \nu_{2 n}, \pi_{2 n}\right) \\
\vdots & \vdots & & \vdots \\
\left(\mu_{n 1}, \nu_{n 1}, \pi_{n 1}\right) & \left(\mu_{n 2}, \nu_{n 2}, \pi_{n 2}\right) & \cdots & \left(\mu_{n n}, \nu_{n n}, \pi_{n n}\right)
\end{array}\right)
$$

is called the intuitionistic judgment matrix, where $\mu_{i j}$ is the degree of membership of alternative $x_{i}$ over alternative $x_{j}, \nu_{i j}$ is the degree of non-membership of alternative $x_{i}$ over alternative $x_{j}$, and $\pi_{i j}$ is the intuitionistic fuzzy index. If the value of $\pi_{i j}$ is large, the degree of hesitancy of $x_{i}$ superior (inferior) to $x_{j}$ is large. In fact, this hesitancy index is a 'regulator'; DMs can modify judgments by changing this index. This means that the degrees of membership and non-membership are included in the range $\left[\mu_{i j}, \mu_{i j}+\pi_{i j}\right]$ and $\left[\nu_{i j}, \nu_{i j}+\pi_{i j}\right]$.

As a consequence, the IFPR $R$ can be equivalently transformed into the membership interval fuzzy preference relation $A$ and non-membership interval fuzzy preference relation $B$ as follows:

$$
\begin{gathered}
A=\left(a_{i j}\right)_{n \times n}=\left[a_{i j l}, a_{i j u}\right]_{n \times n}=\left(\begin{array}{cccc}
{\left[\mu_{11}, \mu_{11}+\pi_{11}\right]} & {\left[\mu_{12}, \mu_{12}+\pi_{12}\right]} & \cdots & {\left[\mu_{1 n}, \mu_{1 n}+\pi_{1 n}\right]} \\
{\left[\mu_{21}, \mu_{21}+\pi_{21}\right]} & {\left[\mu_{22}, \mu_{22}+\pi_{22}\right]} & \cdots & {\left[\mu_{2 n}, \mu_{2 n}+\pi_{2 n}\right]} \\
\vdots & \vdots & & \vdots \\
{\left[\mu_{n 1}, \mu_{n 1}+\pi_{n 1}\right]} & {\left[\mu_{n 2}, \mu_{n 2}+\pi_{n 2}\right]} & \cdots & {\left[\mu_{n n}, \mu_{n n}+\pi_{n n}\right]}
\end{array}\right) \\
B=\left(b_{i j}\right)_{n \times n}=\left[b_{i j l}, b_{i j u}\right]_{n \times n}=\left(\begin{array}{cccc}
{\left[\nu_{11}, \nu_{11}+\pi_{11}\right]} & {\left[\nu_{12}, \nu_{12}+\pi_{12}\right]} & \cdots & {\left[\nu_{1 n}, \nu_{1 n}+\pi_{1 n}\right]} \\
{\left[\nu_{21}, \nu_{21}+\pi_{21}\right]} & {\left[\nu_{22}, \nu_{22}+\pi_{22}\right]} & \cdots & {\left[\nu_{2 n}, \nu_{2 n}+\pi_{2 n}\right]} \\
\vdots & \vdots & & \vdots \\
{\left[\nu_{n 1}, \nu_{n 1}+\pi_{n 1}\right]} & {\left[\nu_{n 2}, \nu_{n 2}+\pi_{n 2}\right]} & \cdots & {\left[\nu_{n n}, \nu_{n n}+\pi_{n n}\right]}
\end{array}\right)
\end{gathered}
$$

Obviously,

$$
\begin{array}{r}
{\left[\mu_{i i}, \mu_{i i}+\pi_{i i}\right]=[0.5,0.5], \mu_{i j}+\left(\mu_{j i}+\pi_{j i}\right)=\left(\mu_{i j}+\pi_{i j}\right)+\mu_{j i}=1, i, j \in N} \\
{\left[\nu_{i i}, \nu_{i i}+\pi_{i i}\right]=[0.5,0.5], \nu_{i j}+\left(\nu_{j i}+\pi_{j i}\right)=\left(\nu_{i j}+\pi_{i j}\right)+\nu_{j i}=1, i, j \in N}
\end{array}
$$


which indicate that both $A$ and $B$ are interval fuzzy preference relations. Therefore the discussion about the equivalent matrices $A$ and $B$ can replace that about the IFPR $R$.

Evidently,

$$
a_{i j l}+b_{i j u}=1, b_{i j l}+a_{i j u}=1
$$

The consistency theorem of an IFPR can be deduced from that of an interval fuzzy preference relation (the IFPR $R$ is transformed into the interval fuzzy preference relations $A$ and $B$ ) as follows:

Theorem 3: Assume that the interval fuzzy preference relations $A=\left(a_{i j}\right)_{n \times n}$ and $B=$ $\left(b_{i j}\right)_{n \times n}$ with coefficients $\theta_{i j}^{A}$ and $\theta_{i j}^{B}$ have the weight vectors $\bar{\omega}^{A}=\left(\bar{\omega}_{1}^{A}, \bar{\omega}_{2}^{A}, \ldots, \bar{\omega}_{n}^{A}\right)^{T}$ and $\bar{\omega}^{B}=$ $\left(\bar{\omega}_{1}^{B}, \bar{\omega}_{2}^{B}, \ldots, \bar{\omega}_{n}^{B}\right)^{T}$ respectively, such that

$$
\begin{aligned}
a_{i j} & =\frac{1}{2}\left(\bar{\omega}_{i}^{A}-\bar{\omega}_{j}^{A}+1\right), i, j \in N \\
b_{i j} & =\frac{1}{2}\left(\bar{\omega}_{i}^{B}-\bar{\omega}_{j}^{B}+1\right), i, j \in N
\end{aligned}
$$

where $\sum_{i=1}^{n} \bar{\omega}_{i}^{A}=1,0 \leq \bar{\omega}_{i}^{A} \leq 1 ; \sum_{i=1}^{n} \bar{\omega}_{i}^{B}=1,0 \leq \bar{\omega}_{i}^{B} \leq 1 ; a_{i j}=\left(1-\theta_{i j}^{A}\right) a_{i j l}+\theta_{i j}^{A} a_{i j u}, \bar{a}_{j i}=$ $\left(1-\theta_{i j}^{A}\right) a_{j i u}+\theta_{i j}^{A} a_{j i l}, i<j ; b_{i j}=\left(1-\theta_{i j}^{B}\right) b_{i j l}+\theta_{i j}^{B} b_{i j u}, \bar{b}_{j i}=\left(1-\theta_{i j}^{B}\right) b_{j i u}+\theta_{i j}^{B} b_{j i l}, i<j$. Then the IFPR $R$ satisfies additive consistency. $\bar{\omega}^{A}$ and $\bar{\omega}^{B}$ are called the weight vectors of $A$ and $B$. $\bar{\omega}^{A}$ is also regarded as the weight vector of $R$ (from a membership perspective).

Regardless of the interval fuzzy preference relations or IFPRs, we assume that the PC judgment $[a, b]$ is an uncertain range and introduce the coefficient $\theta(0 \leq \theta \leq 1)$ into this numerical range to make it a crisp number: namely $[a, b]=(1-\theta) a+\alpha b$. Now that $[a, b]$ is uncertain, we can assume that it obeys a certain distribution. In the next section, we consider that $[a, b]$ is a linear (uniform) uncertainty distribution and discuss the complementary preference relation and priority ranking. Uncertainty theory was introduced by Liu (2007) as a branch of mathematics, and an uncertainty distribution was proposed in order to describe the internal features of uncertain variables. The uncertainty distribution is a carrier of the incomplete information of an uncertain variable. In many cases, it is sufficient to know the uncertainty distribution rather than the uncertain variable itself (Liu, 2015). Uncertain programming, also proposed by Liu (2009), is a type of mathematical programming involving uncertain variables. 


\section{IFPR obeying linear uncertainty distribution and corresponding con- sistency concept}

Definition $7 \mathrm{Liu}$ (2015): Assume that the uncertain variable $\xi$ obeys a distribution such that

$$
\phi(x)=\left\{\begin{array}{lll}
0, & \text { if } & x \leq a \\
\frac{x-a}{b-a}, & \text { if } & a \leq x \leq b \\
1, & \text { if } & x \geq b
\end{array}\right.
$$

Where $a, b$ are specific numbers, and $a<b$. Then, $\xi$ obeys a linear uncertainty distribution within the range $[a, b]$, expressed as $\xi \sim L(a, b)$. Figure 1 shows the linear uncertainty distribution function.

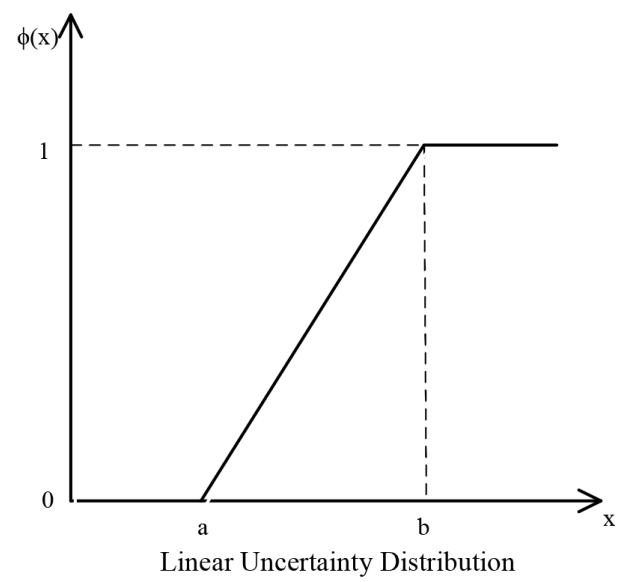

Figure 1: Linear uncertainty distribution function

Definition 8 Liu (2015): Let $\xi$ be an uncertain variable with the linear uncertainty distribution $\phi(x)$. The inverse function $\phi^{-1}(\alpha)$ is called the inverse uncertainty distribution of $\xi$ :

$$
\phi^{-1}(\alpha)=(1-\alpha) a+\alpha b
$$

The inverse linear uncertainty distribution function is shown in Figure 2.

When the interval fuzzy preference relations $A$ and $B$ obey the linear uncertainty distribution,

we use $\tilde{A}$ and $\tilde{B}$ to represent them. Considering the fuzzy preference relation $\tilde{A}=\left(\tilde{a}_{i j}\right)_{n \times n}$, where $\tilde{a}_{i j}=\left[a_{i j l}, a_{i j u}\right]$, if $\tilde{a}_{i j}$ is regarded as a random variable and the value point of $\tilde{a}_{i j}$ is equiprobable within $\left[a_{i j l}, a_{i j u}\right], \tilde{A}=\left(\tilde{a}_{i j}\right)_{n \times n}$ is a linear uncertainty distribution complementary matrix within the range $\left[a_{i j l}, a_{i j u}\right]$. Similar to Theorem 3 , the consistency condition of the IFPR $R$ obeying a linear uncertainty distribution is as follows:

Definition 9: Let the equivalent matrices $\tilde{A}$ and $\tilde{B}$ of the IFPR $R$ obey the linear uncertainty distribution, i.e. $\tilde{a}_{i j} \sim L\left(a_{i j l}, a_{i j u}\right)$ and $\tilde{b}_{i j} \sim L\left(b_{i j l}, b_{i j u}\right)$. If their weight vectors $\omega^{A}=\left(\omega_{1}^{A}, \omega_{2}^{A}, \ldots, \omega_{n}^{A}\right)^{T}$ 


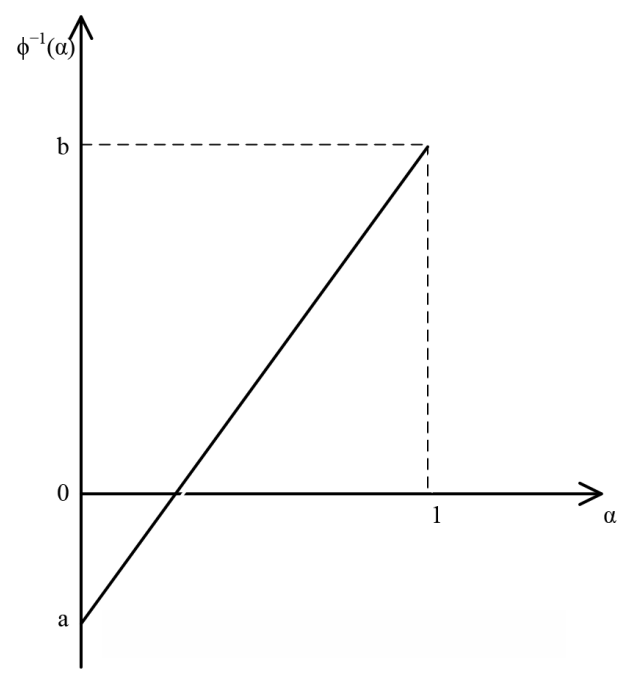

Figure 2: Inverse linear uncertainty distribution function

and $\omega^{B}=\left(\omega_{1}^{B}, \omega_{2}^{B}, \ldots, \omega_{n}^{B}\right)^{T}$ satisfy

$$
\begin{aligned}
& \frac{1}{2}\left(\omega_{i}^{A}-\omega_{j}^{A}+1\right) \sim L\left(a_{i j l}, a_{i j u}\right), i, j \in N \\
& \frac{1}{2}\left(\omega_{i}^{B}-\omega_{j}^{B}+1\right) \sim L\left(b_{i j l}, b_{i j u}\right), i, j \in N
\end{aligned}
$$

respectively, $R$ is called an additive consistency IFPR obeying the linear uncertainty distribution.

\section{Optimal priority modelling with an inconsistency preference relation}

It is difficult for DMs to provide a consistent decision-making matrix under uncertain circumstances because of incomplete information and cognitive behavioural limitations. In this paper, we use $a_{i j}$ to express the actual judgment of DMs, and $a_{i j}^{*}$ is an ideal judgment representing a consistent one. The deviation between $a_{i j}$ and $a_{i j}^{*}$ should satisfy 'the smaller, the better' in evidence. In the following, optimal priority modelling with an inconsistency interval fuzzy preference relation, modelling with an optimal priority IFPR obeying a linear distribution with uncertain chance constraints, and the GDM priority ranking model are presented.

\section{Optimal priority modelling with an inconsistency interval fuzzy preference relation without distribution characteristics}

Previously, if $\bar{A}$ is an inconsistency interval fuzzy preference relation with $\bar{\omega}=\left(\bar{\omega}_{1}, \bar{\omega}_{2}, \ldots, \bar{\omega}_{n}\right)^{T}$ as its weight vector, according to Equation (8), the deviation between $\bar{a}_{i j}$ and $\frac{1}{2}\left(\bar{\omega}_{i}-\bar{\omega}_{j}+1\right)$ should satisfy 'the smaller, the better'; namely, the value of deviation $\varepsilon_{i j}=\left|\bar{a}_{i j}-\frac{1}{2}\left(\bar{\omega}_{i}-\bar{\omega}_{j}+1\right)\right|$ follows 'the smaller, the better'. The programming model for minimizing the deviation on the basis of the 
inconsistency interval fuzzy preference relation is constructed as follows:

$$
\begin{aligned}
& \text { Min } \sum_{i, j \in N, i \neq j} \varepsilon_{i j} \\
& \text { s.t. }\left\{\begin{array}{l}
\left|\bar{a}_{i j}-\frac{1}{2}\left(\bar{\omega}_{i}-\bar{\omega}_{j}+1\right)\right| \leq \varepsilon_{i j} \\
\bar{a}_{i j}=\left(1-\theta_{i j}\right) a_{i j l}+\theta_{i j} a_{i j u} \\
\sum_{i=1}^{n} \bar{\omega}_{i}=1,0 \leq \bar{\omega}_{i} \leq 1,0 \leq \bar{\theta}_{i j} \leq 1 \quad i, j \in N
\end{array} \quad(19-3)\right.
\end{aligned}
$$

The model in Equation (19) has the following equivalent linear form:

$$
\begin{aligned}
& \text { Min } \sum_{i, j \in N, i \neq j} \varepsilon_{i j} \\
& \text { s.t. }\left\{\begin{array}{l}
\bar{a}_{i j}-\frac{1}{2}\left(\bar{\omega}_{i}-\bar{\omega}_{j}+1\right)-\varepsilon_{i j} \leq 0 \\
-\bar{a}_{i j}+\frac{1}{2}\left(\bar{\omega}_{i}-\bar{\omega}_{j}+1\right)-\varepsilon_{i j} \leq 0 \\
\bar{a}_{i j}=\left(1-\theta_{i j}\right) a_{i j l}+\theta_{i j} a_{i j u} \\
\sum_{i=1}^{n} \bar{\omega}_{i}=1,0 \leq \bar{\omega}_{i} \leq 1,0 \leq \bar{\theta}_{i j} \leq 1 \quad i, j \in N
\end{array} \quad(20-2)\right. \\
&
\end{aligned}
$$

\section{Modelling the optimal priority of the IFPR obeying a linear distribution with an uncertain chance constraint}

In the light of the ideology of the chance constraint (Liu and Zhao, 1998), the deviation between the ideal judgment and actual judgment is permitted to be no more than the minimum value to some extent in this paper. However, the probability of this occurring should be no less than a certain level. For the membership matrix $\tilde{A}$ with an inconsistent linear uncertainty distribution, the corresponding priority vector is $\omega^{A}=\left(\omega_{1}^{A}, \omega_{2}^{A}, \ldots, \omega_{n}^{A}\right)^{T}$, assuming that the value of $\tilde{a}_{i j} \sim L\left(a_{i j l}, a_{i j u}\right), i, j \in N$ is independent. Under a certain confidence level, for any value of the stochastic decision variable $\tilde{a}_{i j}$ obeying a linear uncertainty distribution, the deviation between it and $\frac{1}{2}\left(\omega_{i}^{A}-\omega_{j}^{A}+1\right)$ (namely, the value of $\left.\left|\tilde{a}_{i j}-\frac{1}{2}\left(\omega_{i}^{A}-\omega_{j}^{A}+1\right)\right|\right)$ follows 'the smaller, the better'.

The optimal weighting model with the chance constraint is constructed as follows:

$$
\begin{aligned}
& \text { Min } \sum_{i, j \in N, i \neq j} \varepsilon_{i j}^{A} \\
& \text { s.t. }\left\{\begin{array}{l}
M\left\{\left|\tilde{a}_{i j}-\frac{1}{2}\left(\omega_{i}^{A}-\omega_{j}^{A}+1\right)\right| \leq \varepsilon_{i j}^{A}\right\} \geq \alpha \\
\tilde{a}_{i j} \sim L\left(a_{i j l}, a_{i j u}\right) \\
\sum_{i=1}^{n} \omega_{i}^{A}=1,0 \leq \omega_{i}^{A} \leq 1, i \in N
\end{array}\right.
\end{aligned}
$$

where the constraint (21-1) indicates that the probability of the deviation no more than $\varepsilon_{i j}^{A}$ is no less than the certain level $\alpha$, where $\alpha$ is a given value denoting the probability of consistency reaching, called consistency level. Evidently, the higher $\alpha$ is, the consistency level is higher. Here, $\alpha$ takes values of $0.3,0.5,0.7$, and 0.9 , and the objective function minimizes these deviations. 
The optimal priority model with a chance constraint is constructed according to goal programming theory (Liu \& Zhao, 1998). Let $d_{i j}^{A+}$ denote the positive deviation between $\left|\tilde{a}_{i j}-\frac{1}{2}\left(\omega_{i}^{A}-\omega_{j}^{A}+1\right)\right|$ and $\varepsilon_{i j}^{A}$ and $d_{i j}^{A-}$ represent the negative deviation between $\left|\tilde{a}_{i j}-\frac{1}{2}\left(\omega_{i}^{A}-\omega_{j}^{A}+1\right)\right|$ and $\varepsilon_{i j}^{A}$. Evidently, as mentioned above, the deviation satisfies 'the smaller, the better'. The chance-constrained goal programming model is as follows:

$$
\begin{aligned}
& \operatorname{Min} \sum_{i, j \in N, i \neq j} \varepsilon_{i j}^{A}+d_{i j}^{A+}+d_{i j}^{A-} \\
& \text { s.t. } \begin{cases}M\left\{\left|\tilde{a}_{i j}-\frac{1}{2}\left(\omega_{i}^{A}-\omega_{j}^{A}+1\right)\right|-d_{i j}^{A+}+d_{i j}^{A-}=\varepsilon_{i j}^{A}\right\} \geq \alpha & (22-1) \\
\tilde{a}_{i j} \sim L\left(a_{i j l}, a_{i j u}\right) & (22-2) \\
\sum_{i=1}^{n} \omega_{i}^{A}=1,0 \leq \omega_{i}^{A} \leq 1, i \in N\end{cases}
\end{aligned}
$$

In the constraint (22-1), the positive deviation $d_{i j}^{A+}$ and the negative deviation $d_{i j}^{A-}$ satisfy

$$
\begin{array}{r}
M\left\{d_{i j}^{A+} \geq \tilde{a}_{i j}-\frac{1}{2}\left(\omega_{i}^{A}-\omega_{j}^{A}+1\right)-\varepsilon_{i j}^{A}\right\} \geq \alpha \\
M\left\{d_{i j}^{A-} \geq-\tilde{a}_{i j}+\frac{1}{2}\left(\omega_{i}^{A}-\omega_{j}^{A}+1\right)-\varepsilon_{i j}^{A}\right\} \geq \alpha
\end{array}
$$

namely:

$$
\begin{array}{r}
M\left\{\tilde{a}_{i j} \leq \frac{1}{2}\left(\omega_{i}^{A}-\omega_{j}^{A}+1\right)+\varepsilon_{i j}^{A}+d_{i j}^{A+}\right\} \geq \alpha \\
M\left\{-\tilde{a}_{i j} \leq-\frac{1}{2}\left(\omega_{i}^{A}-\omega_{j}^{A}+1\right)+\varepsilon_{i j}^{A}+d_{i j}^{A-}\right\} \geq \alpha
\end{array}
$$

This model is an uncertain optimization model; thus, a genetic algorithm (Holland,1992) can be adopted to obtain an approximate solution. In fact, it can be transformed into a deterministic model according to uncertainty optimization theory.

Lemma 1 Liu (2009): Assume that $h_{1}(x), h_{2}(x), \ldots, h_{n}(x), h_{0}(x)$ are real-valued functions and that $\xi_{1}, \xi_{2}, \ldots, \xi_{g}, g \in N$ are independent linear uncertain variables satisfying uniform distributions $L\left(a_{1}, b_{1}\right), L\left(a_{2}, b_{2}\right), \ldots, L\left(a_{g}, b_{g}\right)$ separately with the regular uncertainty distributions $\phi_{1}, \phi_{2}, \cdots, \phi_{g}$. For any confidence level $\alpha$, the chance constraint has the form:

$$
M\left\{\sum_{i=1}^{n} \xi_{g} h_{g}(x) \leq h_{0}(x)\right\} \geq \alpha, \quad g \in N
$$

holds if and only if

$$
\sum_{g=1}^{n} h_{g}^{+}(x) \phi_{g}^{-1}(\alpha)-\sum_{g=1}^{n} h_{g}^{-}(x) \phi_{g}^{-1}(1-\alpha) \leq h_{0}(x)
$$

holds

If $h_{1}(x), h_{2}(x), \ldots, h_{n}(x)$ are all non-negative, Equation $(28)$ becomes $\sum_{g=1}^{n} h_{g}(x) \phi_{g}^{-1}(\alpha) \leq h_{0}(x)$, If $h_{1}(x), h_{2}(x), \ldots, h_{n}(x)$ are all non-positive, Equation (28) becomes $\sum_{g=1}^{n} h_{g}(x) \phi_{g}^{-1}(1-\alpha) \leq h_{0}(x)$, where $\phi_{g}^{-1}(\alpha)=(1-\alpha) a_{g}+\alpha b_{g}, \phi_{g}^{-1}(1-\alpha)=\alpha a_{g}+(1-\alpha) b_{g}, g \in N$. 
The corresponding forms of the chance constraints in Equations (25) and (26) are:

$$
\begin{aligned}
(1-\alpha) a_{i j l}+\alpha_{i j}^{A} a_{i j u} & \leq \frac{1}{2}\left(\omega_{i}^{A}-\omega_{j}^{A}+1\right)+\varepsilon_{i j}^{A}+d_{i j}^{A+} \\
(1-\alpha)\left(-a_{i j u}\right)+\alpha_{i j}^{A}\left(-a_{i j l}\right) & \leq-\frac{1}{2}\left(\omega_{i}^{A}-\omega_{j}^{A}+1\right)+\varepsilon_{i j}^{A}+d_{i j}^{A-}
\end{aligned}
$$

The optimal goal programming model of the membership fuzzy preference relation $\tilde{A}$ obeying a linear distribution is given as

$$
\begin{array}{ll}
\operatorname{Min} & \sum_{i<j, i, j \in N} \varepsilon_{i j}^{A}+d_{i j}^{A+}+d_{i j}^{A-} \\
\text { s.t. } & \begin{cases}(1-\alpha) a_{i j l}+\alpha_{i j}^{A} a_{i j u} \leq \frac{1}{2}\left(\omega_{i}^{A}-\omega_{j}^{A}+1\right)+\varepsilon_{i j}^{A}+d_{i j}^{A+} & (31-1) \\
(1-\alpha)\left(-a_{i j u}\right)+\alpha_{i j}^{A}\left(-a_{i j l}\right) \leq-\frac{1}{2}\left(\omega_{i}^{A}-\omega_{j}^{A}+1\right)+\varepsilon_{i j}^{A}+d_{i j}^{A-} & (31-2) \\
\sum_{i=1}^{n} \omega_{i}^{A}=1, \omega_{i}^{A} \geq 0, i, j \in N & (31-3)\end{cases}
\end{array}
$$

Analogously, the optimal goal programming model of the non-membership fuzzy preference relation $\tilde{B}$ obeying a linear distribution is given as

$$
\begin{aligned}
\operatorname{Min} & \sum_{i<j, i, j \in N} \varepsilon_{i j}^{B}+d_{i j}^{B+}+d_{i j}^{B-} \\
\text { s.t. } & \begin{cases}(1-\alpha) b_{i j l}+\alpha_{i j}^{B} b_{i j u} \leq \frac{1}{2}\left(\omega_{i}^{B}-\omega_{j}^{B}+1\right)+\varepsilon_{i j}^{B}+d_{i j}^{B+} & (32-1) \\
(1-\alpha)\left(-b_{i j u}\right)+\alpha_{i j}^{B}\left(-b_{i j l}\right) \leq-\frac{1}{2}\left(\omega_{i}^{B}-\omega_{j}^{B}+1\right)+\varepsilon_{i j}^{B}+d_{i j}^{B-} & (32-2) \\
\sum_{i=1}^{n} \omega_{i}^{B}=1, \omega_{i}^{B} \geq 0, i, j \in N\end{cases}
\end{aligned}
$$

Let $x_{i j}=\frac{1}{2}\left(\omega_{i}^{A}-\omega_{j}^{A}+1\right)$ and $y_{j i}=\frac{1}{2}\left(\omega_{j}^{B}-\omega_{i}^{B}+1\right)$. Hence, the following theorems can be obtained:

Theorem 4: The optimal solutions of chance-constrained programming models with membership matrix $\tilde{A}$ and non-membership matrix $\tilde{B}$ of the IFPR $R$ under different probabilities satisfy: $x_{i j}=y_{j i}$, $\varepsilon_{i j}^{A}=\varepsilon_{i j}^{B}, \sum \varepsilon_{i j}^{A}+d_{i j}^{A+}+d_{i j}^{A-}=\sum \varepsilon_{i j}^{B}+d_{i j}^{B+}+d_{i j}^{B-}$; and the optimal objective values are the same.

Proof. Substituting Equation (12) into Equations (32-1) and (32-2),

$$
\begin{aligned}
(1-\alpha)\left(-a_{i j u}\right)+\alpha_{i j}^{B}\left(-a_{i j l}\right) & \leq-\frac{1}{2}\left(\omega_{j}^{B}-\omega_{i}^{B}+1\right)+\varepsilon_{i j}^{B}+d_{i j}^{B+} \\
(1-\alpha) a_{i j l}+\alpha_{i j}^{B} a_{i j u} & \leq \frac{1}{2}\left(\omega_{j}^{B}-\omega_{i}^{B}+1\right)+\varepsilon_{i j}^{B}+d_{i j}^{B-}
\end{aligned}
$$

Owing to $x_{i j}, y_{i j}$ we defined, the forms of Equations (33) and (34) coincide with those of Equations (31-2) and (31-1), the theorem is proved.

Theorem 5: The priority ranking solutions of the equivalent membership matrix $\tilde{A}$ and nonmembership matrix $\tilde{B}$ of the IFPR $R$ with different additive consistency levels derived by chance constrained programming models have an inverse relationship. 
Proof. From Theorem 4, $\frac{1}{2}\left(\omega_{i}^{A}-\omega_{j}^{A}+1\right)=\frac{1}{2}\left(\omega_{j}^{B}-\omega_{i}^{B}+1\right)$, namely, $\omega_{i}^{A}-\omega_{j}^{A}=\omega_{j}^{B}-\omega_{i}^{B}$. Hence for Model (31), if there exist weight vectors $\omega_{\sigma 1}^{A} \geq \omega_{\sigma 2}^{A} \geq \cdots \geq \omega_{\sigma i}^{A} \geq \cdots \geq \omega_{\sigma n}^{A}, i \in N$, because $\omega_{i}^{A}-\omega_{j}^{A}=\omega_{j}^{B}-\omega_{i}^{B}$, the weight vectors of Model (32) satisfy: $\omega_{\sigma 1}^{B} \leq \omega_{\sigma 2}^{B} \leq \cdots \leq \omega_{\sigma i}^{B} \leq \cdots \leq \omega_{\sigma n}^{B}, i \in$ $N$.

\section{Optimal weighting IFPR model with uncertainty chance-constrained linear distri- bution}

Suppose that there are $m(m \in M)$ DMs having given interval uncertain preference relations obeying the linear independent distribution $A^{k}=\left(a_{i j}^{k}\right)=\left[a_{i j l}^{k}, a_{i j u}^{k}\right], k \in M$ in a multi-criteria GDM system, whose corresponding weights are $\omega^{k}, k \in M$. The DMs' group interval uncertain judgment is $A^{*}=\left(a_{i j}^{*}\right)$, where $a_{i j}^{*}=\sum_{k=1}^{m} \omega^{k} a_{i j}^{k}$. Next we prove that $a_{i j}^{*}$ still obeys a linear uncertainty distribution. First, we consider the following lemma.

Lemma 2 Liu (2015): Assume that $\xi_{1}, \xi_{2}$ are independent linear uncertain variables, presented in the form $L\left(a_{1}, b_{1}\right)$ and $L\left(a_{2}, b_{2}\right)$.

Then (1) $\xi_{1}+\xi_{2}$ is also a linear uncertain variable, i.e. $L\left(a_{1}, b_{1}\right)+L\left(a_{2}, b_{2}\right)=L\left(a_{1}+a_{2}, b_{1}+b_{2}\right)$.

(2)The dot product of the linear uncertainty distribution is still a linear uncertain variable, namely, there exist a scalar number $l>0, l \in R$ such that: $l \cdot L(a, b)=L(l a, l b)$.

On the basis of (1) and (2), let $\lambda=\lambda_{1}+\lambda_{2}$; then $\lambda L(a, b)=\left(\lambda_{1}+\lambda_{2}\right) L(a, b)=L\left(\left(\lambda_{1}+\lambda_{2}\right) a,\left(\lambda_{1}+\right.\right.$ $\left.\left.\lambda_{2}\right) b\right)=L\left(\lambda_{1} a+\lambda_{2} a, \lambda_{1} b+\lambda_{2} b\right)=L\left(\lambda_{1} a, \lambda_{1} b\right)+L\left(\lambda_{2} a, \lambda_{2} b\right)=\lambda_{1} L(a, b)+\lambda_{2} L(a, b)$ holds.

According to the addition and multiplication rules of a linear uncertainty distribution and assuming $\omega^{k}$ is unknown, $a_{i j}^{*}=\sum_{k=1}^{m} \omega^{k} a_{i j}^{k}$; then, $a_{i j}^{*} \sim L\left(\sum_{k=1}^{m} \omega^{k} a_{i j l}^{k}, \sum_{k=1}^{m} \omega^{k} a_{i j u}^{k}\right)$.

Theorem 6: The uncertain GDM judgment $a_{i j}^{*}$ obeys a linear uncertainty distribution.

Proof. By Lemma 2, it can be deduced that uncertain GDM judgment $a_{i j}^{*}$ obeys the linear uncertainty distribution.

If the variable $a_{i j}^{*}$ within the linear uncertainty distribution preference relation satisfies additive consistency, $a_{i j}^{*} \sim \frac{1}{2}\left(\hat{\omega}_{i}-\hat{\omega}_{j}+1\right)$ can be obtained. Analogously, the optimal goal programming model with an inconsistency IFPR obeying a linear uncertainty distribution in the GDM problem is given as follows. The optimization model of the membership matrix is expressed as

$$
\begin{aligned}
& \operatorname{Min} \sum_{i, j \in N, i \neq j} \varepsilon_{i j}^{A^{*}} \\
& \text { s.t. }\left\{\begin{array}{l}
M\left\{\left|a_{i j}^{*}-\frac{1}{2}\left(\omega_{i}^{A^{*}}-\omega_{j}^{A^{*}}+1\right)\right| \leq \varepsilon^{A^{*}}\right\} \geq \alpha \\
a_{i j}^{*} \sim L\left(\sum_{k=1}^{m} \omega^{k} a_{i j l}^{k}, \sum_{k=1}^{m} \omega^{k} a_{i j u}^{k}\right) \\
\sum_{i=1}^{n} \omega_{i}^{A^{*}}=1,0 \leq \omega_{i}^{A^{*}} \leq 1, i \in N
\end{array}\right.
\end{aligned}
$$


Moreover, the optimization model of the non-membership matrix is

$$
\begin{aligned}
& \operatorname{Min} \sum_{i, j \in N, i \neq j} \varepsilon_{i j}^{B^{*}} \\
& \text { s.t. }\left\{\begin{array}{l}
M\left\{\left|b_{i j}^{*}-\frac{1}{2}\left(\omega_{i}^{B^{*}}-\omega_{j}^{B^{*}}+1\right)\right| \leq \varepsilon^{B^{*}}\right\} \geq \alpha \quad(36-1) \\
b_{i j}^{*} \sim L\left(\sum_{k=1}^{m} \omega^{k} b_{i j l}^{k}, \sum_{k=1}^{m} \omega^{k} b_{i j u}^{k}\right) \\
\sum_{i=1}^{n} \omega_{i}^{B^{*}}=1,0 \leq \omega_{i}^{B^{*}} \leq 1, i \in N
\end{array}\right.
\end{aligned}
$$

Similar to the process presented in an earlier section, let $x_{i j}^{*}=\frac{1}{2}\left(\omega_{i}^{A^{*}}-\omega_{j}^{A^{*}}+1\right), y_{j i}^{*}=\frac{1}{2}\left(\omega_{i}^{B^{*}}-\right.$ $\left.\omega_{j}^{B^{*}}+1\right)$, the following theorems can be proven.

Theorem 7: If each IFPR satisfies a linear uncertainty distribution in GDM, the collective IFPR also obeys this distribution, and optimal solutions of the chance-constrained programming models with the membership matrix $A^{*}$ and non-membership matrix $B^{*}$ of this IFPR with different additive consistency levels satisfy: $x_{i j}^{*}=y_{j i}^{*}, \sum \varepsilon_{i j}^{A^{*}}=\sum \varepsilon_{i j}^{B^{*}}$; and the optimal objective values are the same.

Theorem 8: The priority ranking solutions of the equivalent membership matrix $A^{*}$ and nonmembership matrix $B^{*}$ of the ideal IFPR $R^{*}$ with different additive consistency levels derived by chance-constrained programming models have an inverse relationship.

The proofs are omitted. The membership weight vector derived by the above-mentioned chanceconstrained programming model is a priority ranking solution of GDM.

\section{Numerical examples}

\section{Case description}

Assume that three DMs $\left(D^{k}, k=1,2,3\right)$ in a decision making system compare four objects $\left(x_{1}, x_{2}, x_{3}, x_{4}\right)$ in a pairwise manner by $\operatorname{IFPRs}\left(R^{k}, k=1,2,3\right)$ as follows:

$$
\begin{array}{r}
R^{1}=\left(\mu_{i j}^{1}, \nu_{i j}^{1}, \pi_{i j}^{1}\right)=\left(\begin{array}{cccc}
(0.5,0.5,0) & (0.3,0.5,0.2) & (0.5,0.4,0.1) & (0.4,0.6,0) \\
(0.5,0.3,0.2) & (0.5,0.5,0) & (0.5,0.3,0.2) & (0.2,0.7,0.1) \\
(0.4,0.5,0.1) & (0.3,0.5,0.2) & (0.5,0.5,0) & (0.3,0.5,0.2) \\
(0.6,0.4,0) & (0.7,0.2,0.1) & (0.5,0.3,0.2) & (0.5,0.5,0)
\end{array}\right) \\
R^{2}=\left(\mu_{i j}^{2}, \nu_{i j}^{2}, \pi_{i j}^{2}\right)=\left(\begin{array}{cccc}
(0.5,0.5,0) & (0.35,0.5,0.15) & (0.2,0.7,0.1) & (0.5,0.4,0.1) \\
(0.5,0.35,0.15) & (0.5,0.5,0) & (0.6,0.2,0.2) & (0.4,0.5,0.1) \\
(0.7,0.2,0.1) & (0.2,0.6,0.2) & (0.5,0.5,0) & (0.3,0.5,0.2) \\
(0.4,0.5,0.1) & (0.5,0.4,0.1) & (0.5,0.3,0.2) & (0.5,0.5,0)
\end{array}\right)
\end{array}
$$




$$
R^{3}=\left(\mu_{i j}^{3}, \nu_{i j}^{3}, \pi_{i j}^{3}\right)=\left(\begin{array}{cccc}
(0.5,0.5,0) & (0.3,0.5,0.2) & (0.5,0.45,0.05) & (0.4,0.5,0.1) \\
(0.5,0.3,0.2) & (0.5,0.5,0) & (0.6,0.1,0.3) & (0.5,0.4,0.1) \\
(0.45,0.5,0.05) & (0.1,0.6,0.3) & (0.5,0.5,0) & (0.35,0.5,0.15) \\
(0.5,0.4,0.1) & (0.4,0.5,0.1) & (0.5,0.35,0.15) & (0.5,0.5,0)
\end{array}\right)
$$

Let $A^{k}, B^{k}, k=1,2,3$ represent the corresponding membership matrix and non-membership matrix of the IFPR $R^{k}$ given by the DM $D^{k}$ and $\omega_{i}^{A k}, \omega_{i}^{B k}, i \in N$ be the priority ranking of the different judgment relations $A^{k}$ and $B^{k}$ made by $D^{k}$. Table 1 summarizes the results for the weight vectors of the membership and non-membership matrices $(\alpha=0.3,0.5,0.7$ and 0.9$)$.

Table 1: Weights of the Membership and Non-membership Matrices

\begin{tabular}{ccccc|ccccc}
\hline$A$ & 0.3 & 0.5 & 0.7 & 0.9 & $B$ & 0.3 & 0.5 & 0.7 & 0.9 \\
\hline$\omega_{1}^{A 1}$ & 0.1835 & 0.1746 & 0.1732 & 0.1732 & $\omega_{1}^{B 1}$ & 0.3158 & 0.3246 & 0.3256 & 0.3254 \\
$\omega_{2}^{A 1}$ & 0.2804 & 0.3254 & 0.3268 & 0.3268 & $\omega_{2}^{B 1}$ & 0.2203 & 0.1754 & 0.1744 & 0.1746 \\
$\omega_{3}^{A 1}$ & 0.1525 & 0.1254 & 0.1268 & 0.1268 & $\omega_{3}^{B 1}$ & 0.3482 & 0.3754 & 0.3744 & 0.3746 \\
$\omega_{4}^{A 1}$ & 0.3835 & 0.3746 & 0.3732 & 0.3732 & $\omega_{4}^{B 1}$ & 0.1158 & 0.1246 & 0.1256 & 0.1254 \\
$\sum \varepsilon_{i j}^{A 1}$ & 0.38 & 0.5 & 0.66 & 0.82 & $\sum \varepsilon_{i j}^{B 1}$ & 0.38 & 0.5 & 0.66 & 0.82 \\
\hline$\omega_{1}^{A 2}$ & 0.1759 & 0.2015 & 0.1968 & 0.1972 & $\omega_{1}^{B 2}$ & 0.3263 & 0.2978 & 0.3027 & 0.304 \\
$\omega_{2}^{A 2}$ & 0.3447 & 0.3515 & 0.3486 & 0.3472 & $\omega_{2}^{B 2}$ & 0.1546 & 0.1478 & 0.1527 & 0.154 \\
$\omega_{3}^{A 2}$ & 0.1658 & 0.1235 & 0.1264 & 0.1278 & $\omega_{3}^{B 2}$ & 0.3331 & 0.3772 & 0.3723 & 0.371 \\
$\omega_{4}^{A 2}$ & 0.3136 & 0.3235 & 0.3264 & 0.3278 & $\omega_{4}^{B 2}$ & 0.186 & 0.1772 & 0.1723 & 0.171 \\
$\sum \varepsilon_{i j}^{A 2}$ & 0.45 & 0.55 & 0.72 & 0.89 & $\sum \varepsilon_{i j}^{B 2}$ & 0.45 & 0.55 & 0.72 & 0.89 \\
\hline$\omega_{1}^{A 3}$ & 0.1625 & 0.1875 & 0.1875 & 0.1875 & $\omega_{1}^{B 3}$ & 0.3375 & 0.3125 & 0.3125 & 0.3125 \\
$\omega_{2}^{A 3}$ & 0.4425 & 0.3875 & 0.3875 & 0.3875 & $\omega_{2}^{B 3}$ & 0.0575 & 0.1125 & 0.1125 & 0.1125 \\
$\omega_{3}^{A 3}$ & 0.0925 & 0.1375 & 0.1375 & 0.1375 & $\omega_{3}^{B 3}$ & 0.4075 & 0.3625 & 0.3625 & 0.3625 \\
$\omega_{4}^{A 3}$ & 0.3025 & 0.2875 & 0.2875 & 0.2875 & $\omega_{4}^{B 3}$ & 0.1975 & 0.2125 & 0.2125 & 0.2125 \\
$\sum \varepsilon_{i j}^{A 3}$ & 0.015 & 0.125 & 0.305 & 0.485 & $\sum \varepsilon_{i j}^{B 3}$ & 0.015 & 0.125 & 0.305 & 0.485 \\
\hline
\end{tabular}

From Table 1, the optimal solutions of the membership and non-membership preference relations obtained by uncertain programming are obviously the same. Moreover, taking IFPR $R_{1}$ as an example, the priority ranking of the membership matrix $A^{1}$ for different consistency levels is $x_{4} \succ x_{2} \succ x_{1} \succ x_{3}$, whereas that of the non-membership matrix $B^{1}$ for different consistency levels is $x_{3} \succ x_{1} \succ x_{2} \succ x_{4}$, illustrating the reverse relationship with $A^{1}$. Thus, we can verify that Theorem 4 and 5 are correct.

Let the weights be $\omega^{1}=0.4, \omega^{2}=0.3$ and $\omega^{3}=0.3$ for these three DMs respectively. The ideal 
preference relation and its priority vector achieved are as follows $(\alpha=0.3,0.5,0.7$ and 0.9$)$ :

$$
R^{*}=\left(\mu_{i j}^{*}, \nu_{i j}^{*}, \pi_{i j}^{*}\right)=\left(\begin{array}{cccc}
(0.5,0.5,0) & (0.315,0.5,0.185) & (0.41,0.505,0.085) & (0.43,0.51,0.06) \\
(0.5,0.315,0.185) & (0.5,0.5,0) & (0.56,0.21,0.23) & (0.35,0.55,0.1) \\
(0.505,0.41,0.085) & (0.21,0.56,0.23) & (0.5,0.5,0) & (0.315,0.5,0.185) \\
(0.51,0.43,0.06) & (0.55,0.35,0.1) & (0.5,0.315,0.185) & (0.5,0.5,0)
\end{array}\right)
$$

Table 2: Priority Vectors of the Ideal Preference Relation

\begin{tabular}{ccccc|ccccr}
\hline$A^{*}$ & 0.3 & 0.5 & 0.7 & 0.9 & $B^{*}$ & 0.3 & 0.5 & 0.7 & 0.9 \\
\hline$\omega_{1}^{*}$ & 0.1693 & 0.1596 & 0.1608 & 0.1607 & $\omega_{1}^{*}$ & 0.3308 & 0.3403 & 0.339 & 0.3389 \\
$\omega_{2}^{*}$ & 0.3255 & 0.3446 & 0.3458 & 0.3457 & $\omega_{2}^{*}$ & 0.1744 & 0.1553 & 0.154 & 0.1539 \\
$\omega_{3}^{*}$ & 0.1545 & 0.1554 & 0.1542 & 0.1543 & $\omega_{3}^{*}$ & 0.3455 & 0.3447 & 0.346 & 0.3461 \\
$\omega_{4}^{*}$ & 0.3507 & 0.3404 & 0.3392 & 0.3393 & $\omega_{4}^{*}$ & 0.1493 & 0.1597 & 0.161 & 0.1611 \\
$\sum \varepsilon_{i j}^{*}$ & 0.1875 & 0.2825 & 0.4515 & 0.6205 & $\sum \varepsilon_{i j}^{*}$ & 0.1875 & 0.2825 & 0.4515 & 0.6205 \\
\hline
\end{tabular}

From Table 2, the optimal solutions of the ideal membership and non-membership preference relations obtained by uncertain programming are the same. Meanwhile, with the same confidence level, the rankings of the two preference relations have an inverse relationship. Thus, we can verify that Theorem 7 and 8 are correct. And the group decision priority ranking can be achieved with different confidence(consistency) levels.

\section{Analysis of the results}

Computation results are presented in chart form. Figure 3 shows the change of relationships between the minimum sum of the deviation derived from chance constrained programming models based on four membership preference relations $A^{1}, A^{2}, A^{3}, A^{*}$ and the consistency level $\alpha$. In this Figure, the minimum deviation increases as consistency level increases for both individual preference relation and collective group preference relation. The higher confidence level indicates more stringent conditions for realizing an ideal judgment. In this circumstance, the consistency can only be guaranteed if the deviation between the ideal judgment and the actual judgment can be increased (namely, the actual judgment deviation threshold is permitted to increase).

Hence, the chance constraint proposed in this paper can be regarded as an effective method for controlling the degree of consistency of preference relations. 


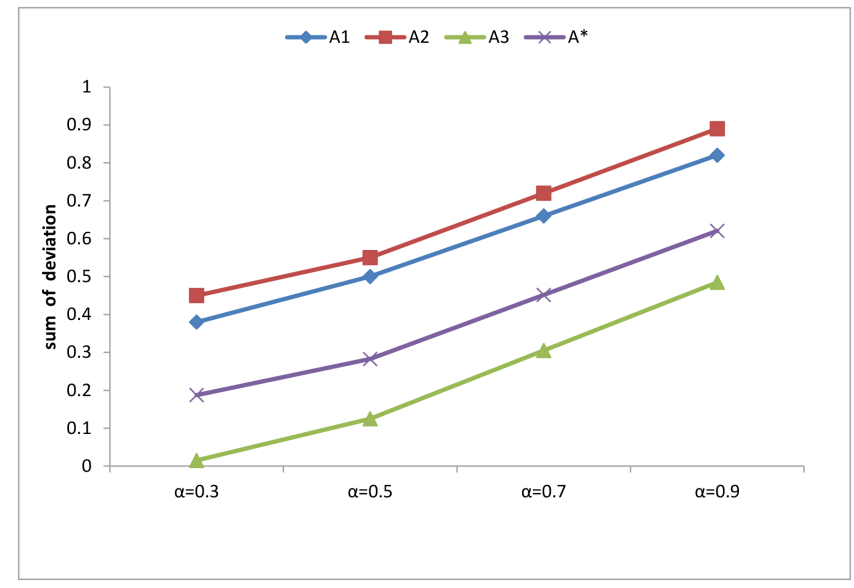

Figure 3: Relationships between the minimum sum of the deviation and the consistency level

\section{Conclusion}

This paper investigates the optimal ranking models of individual and group IFPRs obeying a linear uncertainty distribution; for a certain confidence level, the equivalent deterministic programming models contribute to obtaining the priority weights of the alternatives by exploring the equivalence relations of the IFPR and the interval membership and non-membership fuzzy preference relations based on additive consistency, thereby reducing the cost of stochastic simulation for uncertain programming. Moreover, the introduction of the chance-constrained uncertainty distribution variables based on additive consistency realizes the more reasonable simulation of the uncertainty and fuzziness of a real environment. The contributions of this study are as follows:

(1) An interval fuzzy preference relation equivalent to an IFPR obeying a linear uncertainty distribution is defined, and uncertain programming models with a chance constraint are developed to study the ranking of the alternatives in the IFPR.

(2) The deviation between the ideal consistency judgment and the actual judgment is proposed, and the flexibility of consistency realization can be controlled by adjusting the consistency level. Moreover, different values of $\alpha$ can be regarded as different risk decision-making levels.

Apart from linear uncertainty distribution model, study can consider other distribution such as uncertain normal distribution. In addition, this research could be extended to consensus decisionmaking models based on multiplicative consistency IFPR. 


\section{Acknowledgements}

The work in this paper was supported by the National Natural Science Foundation of China (71571104, 71171115, 70901043), Qing Lan Project, the Six Talent Peaks Project in Jiangsu Province (2014-JY-014), the Project Funded by the Priority Academic Program Development of Jiangsu Higher Education Institutions and the Natural Science Foundation of Jiangsu, China (grant No. BK20141481). And the paper was also supported by the Postgraduate Research \& Practice Innovation Program of Jiangsu Province (KYCX17_0905).

\section{References}

Atanassov K T (1986). Intuitionistic fuzzy sets. Fuzzy Sets and Systems 20: 89-97

Behret H (2014). Group decision making with intuitionistic fuzzy preference relations. Knowledge-Based Systems 70: 33-43.

Chen SM, Cheng SH and Lin TE (2015). Group decision making systems using group recommendations based on interval fuzzy preference relations and consistency matrices. Information Sciences 298: 555-567.

Chen SM, Lin TE and Lee LW (2014). Group decision making using incomplete fuzzy preference relations based on the additive consistency and the order consistency. Information Sciences 259: 1-15.

Chu JF, Liu XW, Wang YM and Chin KS (2016). A group decision making model considering both the additive consistency and group consensus of intuitionistic fuzzy preference relations. Computers $\mathbb{E}$ Industrial Engineering 101: 227-242.

Dong YC, Li CC, Chiclana F and Herrera-Viedma E (2016). Average-case consistency measurement and analysis of interval-valued reciprocal preference relations. Knowledge-Based Systems 114: 108-117.

Gong ZW (2011). Theory, method and application of uncertain fuzzy judgment matrix. Science Press: 96-100.

Gong ZW, Xu XX, Zhang HH, Ozturk UA, Herrera-Viedma E and Xu C (2015). The consensus models with interval preference opinions and their economic interpretation. Omega 55: 81-90.

Gong ZW, Zhang HH, Forrest J, Li Lianshui and Xu Xiaoxia (2015). Two consensus models based on the minimum cost and maximum return regarding either all individuals or one individual. European Journal of Operational Research 240(1):183-192.

Holland J H (1992). Adaptation in natural and artificial systems. MIT Press.

Hu M, Ren P, Lan J, Wang J and Zheng W (2014). Note on "Some models for deriving the priority weights from interval fuzzy preference relations". European Journal of Operational Research 237: 771-773.

Jin FF, Ni ZW, Chen HY and Li YP (2016a). Approaches to group decision making with intuitionistic fuzzy preference relations based on multiplicative consistency. Knowledge-Based Systems 97: 48-59.

Jin FF, Ni ZW, Chen HY and Li YP (2016b). Approaches to decision making with linguistic preference relations based on additive consistency. Applied Soft Computing 49: 71-80. 
Ke H and Liu BD (2007). Project scheduling problem with mixed uncertainty of randomness and fuzziness. European Journal of Operational Research 183(1):135-147.

Krejčí J (2017). On additive consistency of interval fuzzy preference relations. Computers 6 Industrial Engineering 107: 128-140.

Liao HC and Xu ZS (2014). Priorities of intuitionistic fuzzy preference relation based on multiplicative consistency. IEEE Transactions on Fuzzy Systems 22(6): 1669-1681.

Liao HC, Xu ZS, Zeng XJ and Xu DL (2016). An enhanced consensus reaching process in group decision making with intuitionistic fuzzy preference relations. Information Sciences 329(C): 274-286.

Liu BD (2007). Uncertainty Theory, 2nd edn, Springer-Verlag: Berlin.

Liu BD (2009). Uncertainty Theory. UTLAB. Springer-Verlag: Berlin.

Liu BD (2015). Uncertainty Theory. Uncertainty Theory Laboratory. Springer-Verlag: Berlin.

Liu BD and Zhao RQ (1998). Stochastic programming and fuzzing programming. Tsinghua University Press: 74-94.

Liu F, Zhang WG and Shang YF (2016). A group decision-making model with interval multiplicative reciprocal matrices based on the geometric consistency index. Computers $\&$ Industrial Engineering 101: 184-193.

Liu F, Zhang WG and Zhang LH (2014). Consistency analysis of triangular fuzzy reciprocal preference relations. European Journal of Operational Research 235 (3):718-726.

Liu X, Pan Y, Xu Y and Yu S (2012). Least square completion and inconsistency repair methods for additively consistent fuzzy preference relations. Fuzzy Sets and Systems 198: 1-19.

Meng FY, An QX and Chen XH (2016). A consistency and consensus-based method to group decision making with interval linguistic preference relations. Journal of the Operational Research Society 67(11): 1419-1437.

Omidi F, Abbasi B and Nazemi A (2017). An efficient dynamic model for solving a portfolio selection with uncertain chance constraint models. Journal of Computational and Applied Mathematics 319: 43-55.

Ouyang Y and Pedrycz W (2016). A new model for intuitionistic fuzzy multi-attributes decision making. European Journal of Operational Research 249(2): 677-682.

Suo C, Li YP, Wang CX and Yu L (2017). A type-2 fuzzy chance-constrained programming method for planning Shanghai's energy system. International Journal of Electrical Power $\&$ Energy Systems 90: 37-53. Tan CQ, Yi WT and Chen XH (2015). Generalized intuitionistic fuzzy geometric aggregation operators and their application to multi-criteria decision making. Journal of the Operational Research Society 66(11): 1919-1938.

Tan X, Gong ZW, Chiclana F and Zhang N (2017). Consensus modeling with cost chance constraint under uncertainty opinions. Applied Soft Computing

Tanino, T (1984). Fuzzy preference orderings in group decision making. Fuzzy Sets and Systems 12(2): $117-131$.

Wang J, Lan JB, Ren P and Luo YY (2012). Some programming models to derive priority weights from additive interval fuzzy preference relation. Knowledge-Based Systems 27: 69-77. 
Wang ZJ (2013). Derivation of intuitionistic fuzzy weights based on intuitionistic fuzzy preference relations. Applied Mathematical Modelling 37(9): 6377-6388.

Wang ZJ (2015). Uncertainty index based consistency measurement and priority generation with interval probabilities in the analytic hierarchy process. Computers $\&$ Industrial Engineering 83: 252-260.

Wang ZJ (2016). A two-stage linear goal programming approach to eliciting interval weights from additive interval fuzzy preference relations. Soft Computing 20: 2721-2732.

Wang ZJ and Li KW (2015). A multi-step goal programming approach for group decision making with incomplete interval additive reciprocal comparison matrices. European Journal of Operational Research 242(3): 890-900.

Wang ZJ and Li KW (2016). Group decision making with incomplete intuitionistic preference relations based on quadratic programming models. Computers \& Industrial Engineering 93: 162-170.

Wang ZJ and Tong XY (2016). Consistency analysis and group decision making based on triangular fuzzy additive reciprocal preference relations. Information Sciences 361: 29-47.

Wu J and Chiclana F (2014). Multiplicative consistency of intuitionistic reciprocal preference relations and its application to missing values estimation and consensus building. Knowledge-Based Systems 71: 187-200. Wu ZB and Xu JP (2016). Managing consistency and consensus in group decision making with hesitant fuzzy linguistic preference relations. Omega 65: 28-40.

Xu YJ, Li KW and Wang HM (2014). Consistency test and weight generation for additive interval fuzzy preference relations. Soft Computing 18(8): 1499-1513.

Xu ZS (2007). Intuitionistic preference relations and their application in group decision making. Information sciences 177(11): 2363-2379.

$\mathrm{Xu}$ ZS and Xia MM (2014). Iterative algorithms for improving consistency of intuitionistic preference relations. Journal of the Operational Research Society 65(5): 708-722.

Zadeh, L. A (1965). Fuzzy sets. Information and Control 8(3): 338-356.

Zhang HM (2015). A consistency model for group decision making problems with interval multiplicative preference relations. Applied Soft Computing 34: 60-71.

Zhang HM (2016). A goal programming model of obtaining the priority weights from an interval preference relation. Information Sciences 354: 197-210.

Zhang N, Gong ZW and Chiclana F (2017). Minimum Cost Consensus Models based on Random Opinions. Expert Systems with Applications $\mathbf{8 9}$.

Zhang Z (2016). Deriving the priority weights from incomplete hesitant fuzzy preference relations based on multiplicative consistency. Applied Soft Computing 46: 37-59.

Zhang Z and Guo CH (2017). Deriving priority weights from intuitionistic multiplicative preference relations under group decision-making settings. Journal of the Operational Research Society 68(2): 1-18.

Zhang Z, Wang C and Tian X (2015). Multi-criteria group decision making with incomplete hesitant fuzzy preference relations. Applied Soft Computing 36: 1-23.

Zhao M, Ma XY and Wei DW (2017). A Method Considering and Adjusting Individual Consistency and Group Consensus for Group Decision Making with Incomplete Linguistic Preference Relations. Applied Soft 
Computing 54: 322-346.

Zhu B and Xu ZS (2013). Hesitant fuzzy Bonferroni means for multi-criteria decision making. Journal of the Operational Research Society 64(12): 1831-1840. 